GRADIATION\&APPLICATIONS

ISSN 2466-4294 (online) | rad-journal.org

Vol. 2 | Issue 1 | pp. 14 - 19

doi: 10.21175/RadJ.2017.01.004

Original research paper

\title{
DEVELOPMENT OF BREAST SOFTWARE PHANTOM DEDICATED FOR RESEARCH AND EDUCATIONAL PURPOSES*
}

\author{
Kristina Bliznakova** \\ Laboratory of Computer Simulations in Medicine, Technical University of Varna, Varna, Bulgaria
}

\begin{abstract}
Physical test phantoms are valuable tools in the assessment of novel breast imaging techniques. It is important that they reproduce the breast characteristics as close as possible. This paper presents a methodology for the creation of a software breast phantom that produces a structured background in both two dimensional mammography and breast tomosynthesis. The phantom is composed of spherical objects different in size placed in a semi-cylindrical container. This software breast phantom is used to further improve the existing physical phantom in terms of use of other more appropriate materials or a structure presentation different than the use of spheres. For this purpose, a simulation platform that allows the creation of software versions of the physical phantom with parameters (sizes, shapes, materials) easily adjusted by the user, was developed. Besides the research aspects of the computational phantom, the software tool has turned out to be very useful application for the training of Medical Physics Experts. Specifically, the tool has been used in the training of the participants of module 5 "The use of physical and virtual anthropomorphic phantoms for image quality and patient dose optimization" from the EUTEMPE-RX project that is related to the qualification of Medical Physics Experts in Diagnostic and Interventional Radiology.
\end{abstract}

Key words: Software breast phantom, educational and research purposes

\section{INTRODUCTION}

Physical test breast phantoms are valuable tools in the assessment of novel breast imaging techniques. It is important that they reproduce the breast characteristics as close as possible. Such characteristics are for instance the fractal dimension and the power law exponent $\beta$ of mammographic projection images, as well as other parameters like first order, second order or higher order statistical parameters. One such example is the UPenn breast phantom which provides both software and physical realisation [1]. It is also important to reproduce the imaging characteristics in three-dimensional imaging setups.

The LUCMFR group at the Department of Radiology, Catholic University of Leuven has developed a physical phantom [2] that produces a structured background in both two dimensional (2D) mammography and breast tomosynthesis. This physical breast phantom is based on the work of Gang et al. [3] who showed that equal volumes of differently sized acrylic spheres provide a fractal dimension of 3 as well as a power law exponent $\beta$ equal to 3 . The LUCMFR phantom consists of acrylic spheres placed in an acrylic container filled either with air or with water. This phantom has been evaluated on a Siemens mammographic system with tomosynthesis option, showing results for the power law exponent in the range of the exponents measured in patient data [2].

To improve further the existing physical phantom in terms of inclusion of breast lesions, use of other more appropriate materials or structure presentation different than the use of spheres, a computer-based simulation platform was developed that allows the creation of software versions of this phantom with parameters like sizes of container and spheres, and material characteristics, which parameters can be easily adjusted by the user. This work presents the use of this software platform to generate software phantoms, dedicated for breast imaging research as well as for educational purposes.

\section{MATERIALS AND METHODS}

\subsection{Physical phantom description}

The physical phantom developed at LUCMFR is composed of two main parts: an acrylic semi-cylinder container of thickness $58 \mathrm{~mm}$ and diameter of 200 $\mathrm{mm}$, and equal volumes of acrylic spheres of six different diameters: $15.88 \mathrm{~mm}, 12.70 \mathrm{~mm}, 9.52 \mathrm{~mm}$, $6.35 \mathrm{~mm}, 3.18 \mathrm{~mm}$ and $1.58 \mathrm{~mm}$ [2].

The spherical objects are placed within the semicircular container which resembles a compressed * The paper was presented at the Fourth International Conference on Radiation and Applications in Various Fields of Research
(RAD 2016), Niš, Serbia, 2016.

**kristina.bliznakova@gmail.com 
breast. As an alternative to spheres placed in air, the space between the spherical objects can be also filled with water, a material with $\mathrm{x}$-ray properties close to these of the real breast tissue. The later phantom is shown in Figure 1. This physical phantom is characterized with air bubbles which were visible at the upper side of the phantom because of the practical difficulties in completely filling the phantom with water.

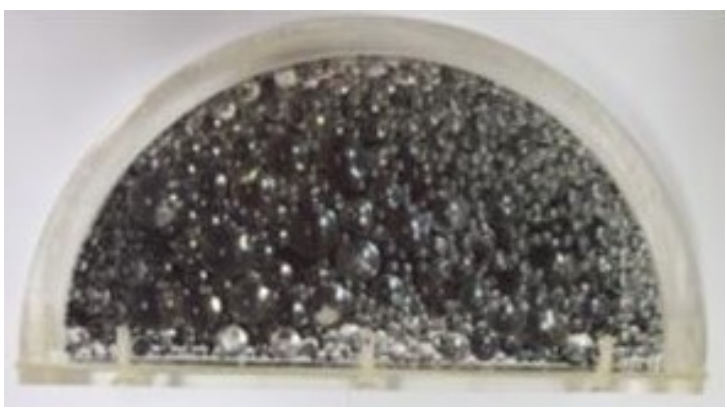

Figure 1. A photograph of the physical phantom

\subsection{Software phantom description}

The software phantom is constructed as composed of two main parts: a semi-cylinder container and spheres of different diameters. The container may be modelled as a set of four semi-cylinders and a slab object (approximating the sidewall) as shown in Figure 2a. The top and the base of the phantom however may be included into the external semi-cylinder. The complete container is then reduced to two semicylinders and one slab object as shown in Figure 2b.
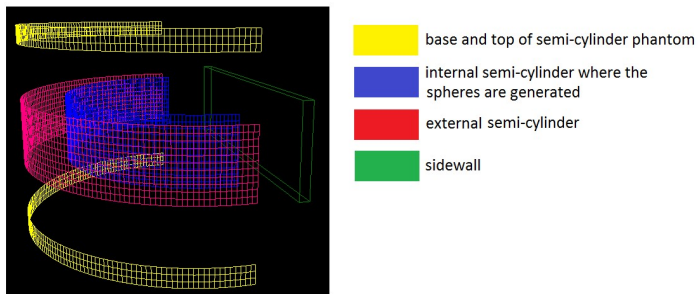

(a)

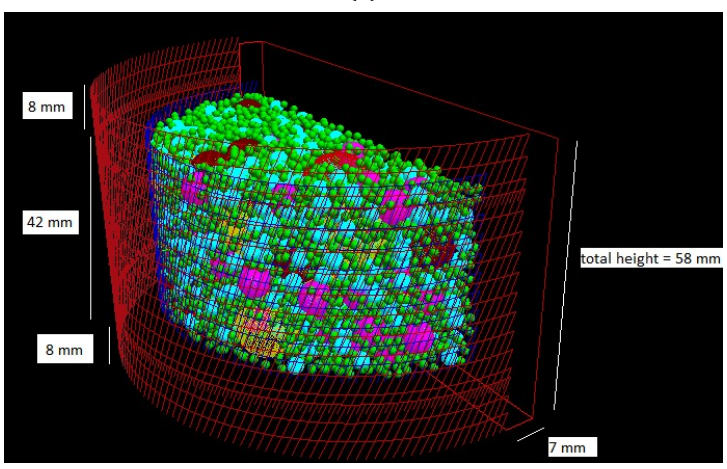

(b)

Figure 2. The software breast phantom (a) external shape components; (b) the complete breast phantom
The software phantom is generated by using the LUCMFRGen tool that is designed to generate phantoms similar to the one shown in Figure 2 and reproducing as close as possible the physical one shown in Figure 1. A screenshot from this tool is shown in Figure 3. It is featured by two main fields: one of the fields is dedicated to the design of the container: dimensions (wall thickness, diameter and height) and material characteristics, while the second field is dedicated to the spherical objects. For instance, the phantom setup shown in Figure 3 corresponds to the creation of a software breast phantom with the same parameters as the physical one (Figure 1). The statistics, shown at the bottom of the platform, indicates that the total number of generated spherical objects is 33376 , as 29 of these are spheres with a diameter of $15.88 \mathrm{~mm}$.

The software application samples randomly the location of the spherical objects within the internal semi-cylinder, starting from the spheres with largest diameter. The algorithm also verifies that the sampled sphere does not have intersection with other spheres at the sampled position.

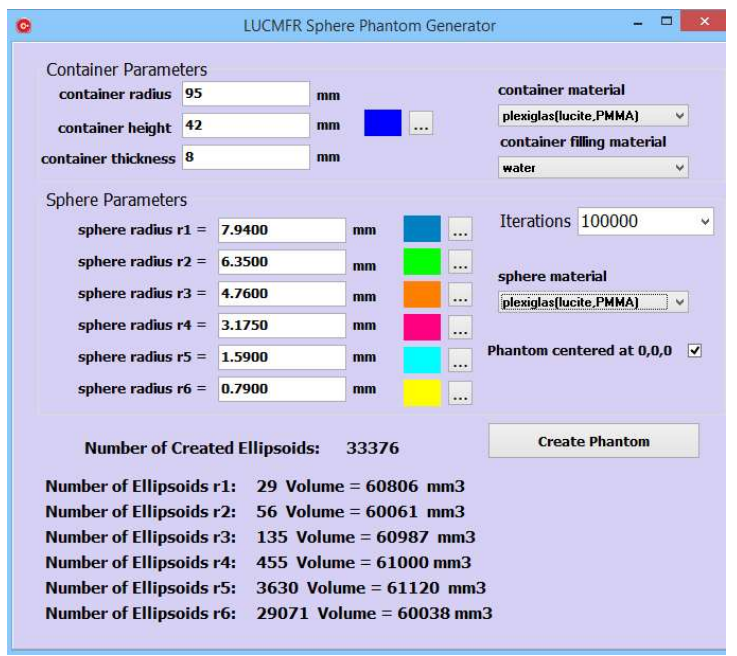

Figure 3. A screenshot from the software application LUCMFRGen showing the design of software breast phantom with similar characteristics to the physical breast phantom

A major advantage of this software application is the possibility to create phantoms with different dimensions and content, as well as the possibility to assign various material characteristics to the simulated objects.

\section{ApPLICATIONS}

\subsection{Research}

The software platform was used to study the appearance of simulated planar images of generated breast phantoms with different characteristics. For this purpose, software breast phantoms which were replica of the physical phantom, shown in Figure 1, were created by following the setup shown in Figure 3. 


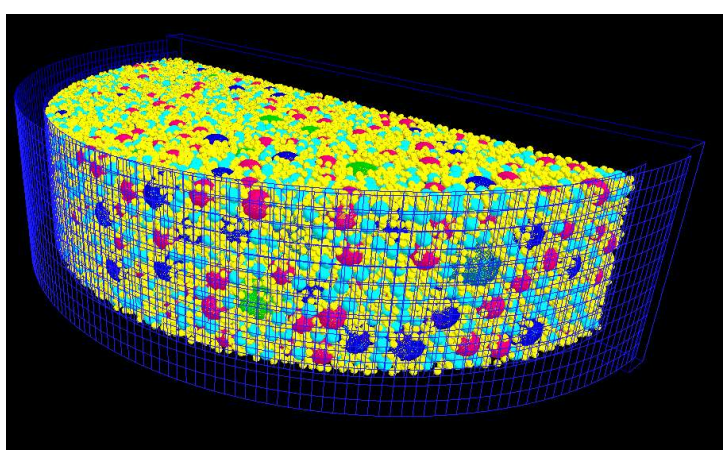

Figure 4. The software phantom: external shape and spherical objects

These phantoms, one of them is shown in Figure 4, are composed of two main parts: a semi-cylinder container of thickness $58 \mathrm{~mm}$ and diameter $200 \mathrm{~mm}$, and equal volumes of spheres of six different diameters: $15.88 \mathrm{~mm}, 12.70 \mathrm{~mm}, 9.52 \mathrm{~mm}, 6.35 \mathrm{~mm}$, $3.18 \mathrm{~mm}$ and $1.58 \mathrm{~mm}$. The total number of generated spherical objects was 33376. As in the physical phantom, the total volume of each different sphere type was set to be equal (approximately $60806 \mathrm{~mm}^{3}$ ).

The three phantoms were: the phantom shown in Figure 4, made of (i) container, filled with air and acrylic spherical objects; (ii) container filled with water and acrylic spheres; and (iii) container filled with water, containing the polyethylene spheres.

Planar x-ray projection images of the software phantoms were obtained with the XRayImagingSimulator [4]. Images were simulated for monochromatic x-ray beam with energy of $19 \mathrm{keV}$. Scatter and detector responses were not simulated. Distances from the source to the breast support table, where the phantom was placed and to the detector surface were $600 \mathrm{~mm}$ and $650 \mathrm{~mm}$, respectively. The size of the images and their resolution was 2500 pixels and $0.10 \mathrm{~mm}$, respectively in each direction.

Figure 5 shows simulated planar images of the first software breast phantom (Figure $5 \mathrm{a}$ ), second software phantom with the water filling material (Figure $5 \mathrm{c}$ ) and the third software breast phantom with the low density polyethylene spherical objects (Figure 5e). Planar images of the first two phantoms are visually compared to experimental ones acquired from the physical phantom filled with water and air (Figure 5b,d). Real images are produced with Siemens Inspiration system, with distances from the source to the breast support table and to the detector equal to $633 \mathrm{~mm}$ and 650 $\mathrm{mm}$.

The comparison of simulated and real x-ray images shows a very good visual appearance of produced simulated 2D mammographic backgrounds. The simulated image from the third virtual phantom with the low density polyethylene spherical objects shows the flexibility of the platform to design different breast phantoms and to obtain simulated images of these phantoms at almost no cost. Computer simulations using such phantom models demonstrate great potential to optimize imaging parameters of new breast imaging modalities.

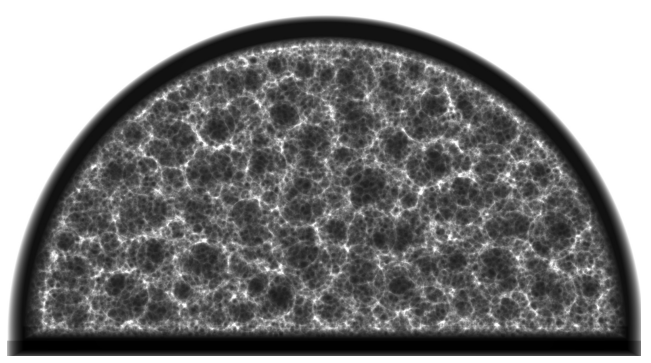

(a)

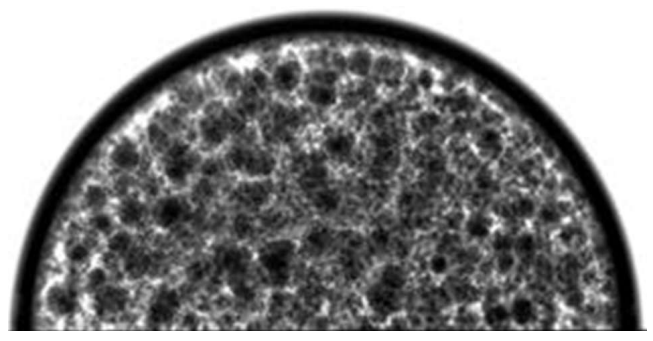

(b)

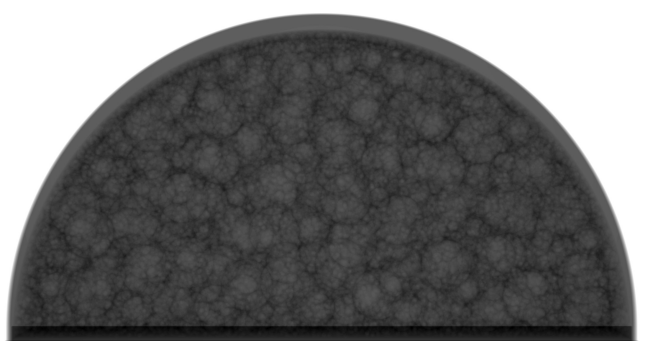

(c)

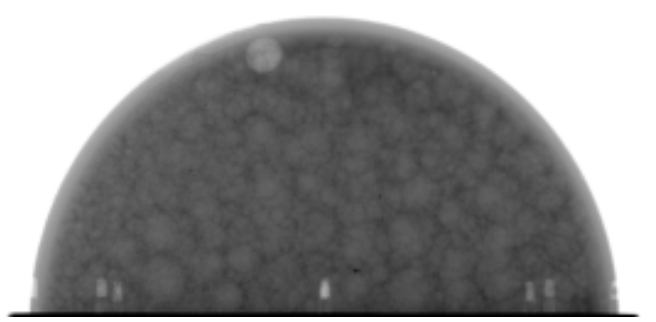

(d)

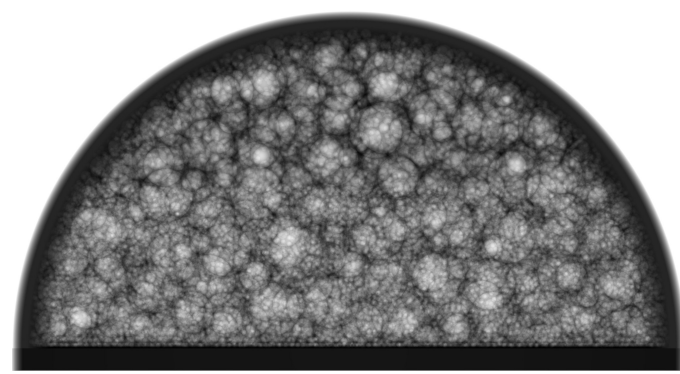

(e)

Figure 5. Comparison of simulated (a, c) and real (b, d) planar intensity images from software and physical breast phantoms filled with acrylic spheres placed in air (a, b) and water (c, d).

In (e) simulated $x$-ray image of a breast phantom with spheres made of low density $\left(0.91 \mathrm{~g} \mathrm{~cm}^{-3}\right)$ polyethylene material 


\subsection{Education}

The LUCMFRGen software tool has turned out to be very useful application for training of the Medical Physics Experts. Specifically, the tool has been used in the training of the participants of module 5 "The use of physical and virtual anthropomorphic phantoms for image quality and patient dose optimization" from the EUTEMPE-RX project (European Training and Education for Medical Physics Experts in Radiology) [5]. In short, the EUTEMPE-RX project aims at the development of a platform devoted to the education of Medical Physics Experts [6] in Radiology. The developed platform provides a model training scheme that allows the medical physicist in Diagnostic and Interventional Radiology (D\&IR) to reach a high level of knowledge, skills and competences, described in [6]. The partners within the EUTEMPE-RX project designed 12 modules by using a blended learning scheme. The Anthropomorphic Phantoms was the fifth module from the EUTEMPE-RX module held at the Technical University of Varna, 7-13 September 2015. The main goals of the module are to familiarize the participants with the role of the physical and virtual anthropomorphic phantoms and the possibility of performing virtual (phantom based) clinical trials using existing and new D\&IR technologies. Participants were encouraged to develop skills for the design and evaluation of anthropomorphic phantoms, as well as to design, manage, implement and evaluate virtual clinical studies with such phantoms, and to discuss and interpret the results of the virtual studies.

A screen shot of the online material for this module is shown in Figure 6. As it can be seen, the first page lists the "syllabus" and/or "the learning goals".

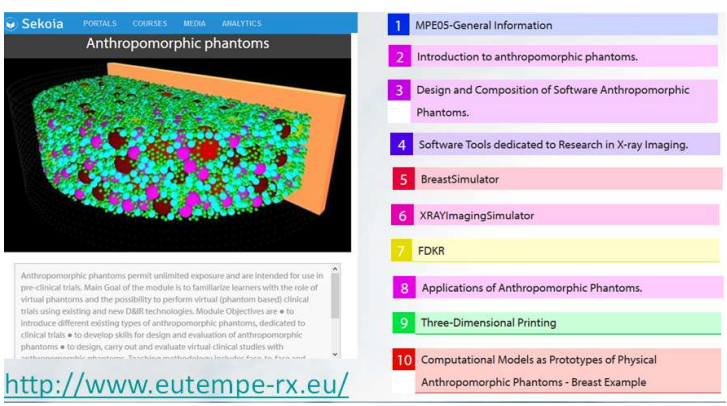

Figure 6. A screenshot from the online e-learning Sekoia platform depicting the module "Anthropomorphic Phantoms" under development

The online phase was performed six weeks before the face-to-face part. The face-to-face component lasted for a period of 1 week, organized in a mixed format that included mainly computer-based exercises (Figure 7). The teaching approach included two lectures per day (each $45 \mathrm{~min}$ ), tutorials, practical sessions and project work.

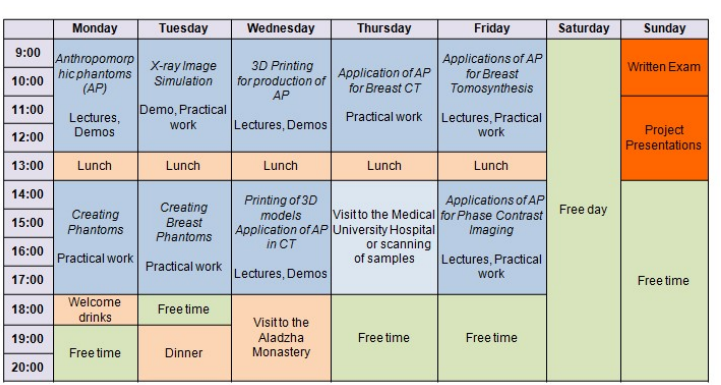

Figure 7. The face-to-face program of the "Anthropomorphic Phantoms" module implemented in Varna, 7-13 September 2015

Three out of nine work projects included the creation of software breast phantom by using the LUCMFRGen software tool. The participants designed themselves breast phantoms using some preliminary requirements like size and content of the phantoms. Consequently, they used the generated phantoms in virtual studies which aimed at testing and optimising advanced breast imaging techniques: breast tomosynthesis, dual energy tomosynthesis, as well as phase contrast imaging. Two examples of such work projects and their implementation are presented bellow.

Work Project Example 1: Using computational breast phantom, perform a virtual study to determine the potential of dual-energy (DE) imaging for detectability of breast abnormalities, compared to conventional mammography.

For this purpose, the participants used the LUCMFRGen software generator to design a compressed breast phantom from an acrylic material with radius $40 \mathrm{~mm}$ and height $35 \mathrm{~mm}$, as shown in Figure 8a. The software tool created 2246 water spheres of diameter randomly sampled between 1.0 $\mathrm{mm}$ and $15.0 \mathrm{~mm}$. In addition, five spheres of size of 1 $\mathrm{mm}$ to $3 \mathrm{~mm}$ were randomly selected by the participants and assigned to have the material $\mathrm{CaCO}_{3}$. The two breast masses were modelled as spherical objects of radius $3 \mathrm{~mm}$ and $6 \mathrm{~mm}$ and attenuation material that corresponded to breast mass tissue: $75 \%$ glandular and $25 \%$ adipose tissue and $100 \%$ glandular tissue, respectively. The phantom was used with the $X$ RayImagingSimulator [4] to generate planar x-ray images for the following incident monochromatic beams: $17 \mathrm{keV}, 23 \mathrm{keV}, 45 \mathrm{keV}, 60 \mathrm{keV}$ and $75 \mathrm{keV}$. Poisson noise was added to the ideal planar images assuming $6 \mathrm{mGy}$ surface entrance dose. Dual-energy images were generated from the subtraction of the noisy projections and contrast-to-noise ratio was used to analyse the detection performance of the simulated lesions. 
The participants studied 6 dual-energy combinations of low and high energy images. Based on contrast-to-noise ratio results as well as visual comparison between six DE images, they found that the optimal low-high energy configuration is $17 \mathrm{keV}$ and $60 \mathrm{keV}$. Dual-energy images were superior to $2 \mathrm{D}$ mammography for lesion detection as the background tissue was eliminated from the image. All simulated small calcifications were well differentiated in both DE and conventional mammography. On opposite, breast masses, which have similar attenuation coefficient to background tissue, were only visible in DE images.

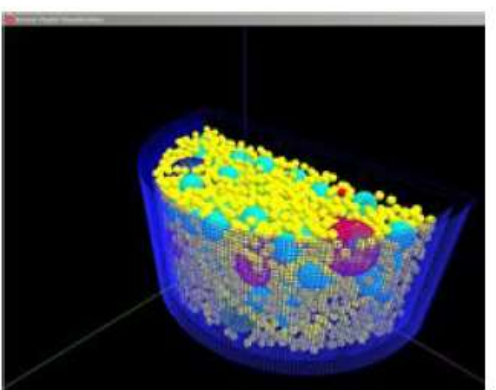

(a)

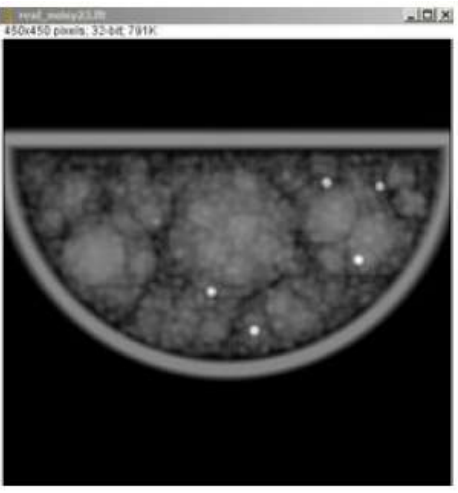

(b)

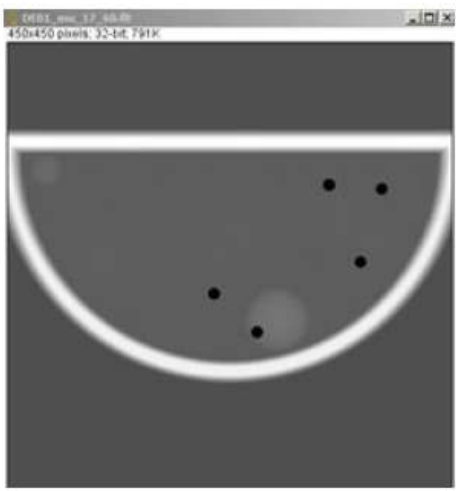

(c)

Figure 8. Work Project Example 1: images are taken from the project work of participants, who performed the face-to-face phase of the "Anthropomorphic Phantoms" module at the Technical University of Varna, Bulgaria, 7-13 September 2015. (a) generated breast software phantom, (b) simulated conventional mammography at $17 \mathrm{keV}$, (c) dual-energy image obtained from simulated planar images for $17 \mathrm{keV}$ and $60 \mathrm{keV}$.

Work Project Example 2: The goal of this work project was to perform a virtual study to determine the potential of breast tomosynthesis compared to conventional mammography for detectability of breast abnormalities using a computational structured phantom.

For this work, the participants generated the breast phantom shown in Figure 9a. The compressed breastshaped container was of size $120 \mathrm{~mm} \times 60 \mathrm{~mm} \times 35$ mm randomly filled with 1430 spheres with different size: radii of $7.94 \mathrm{~mm} ; 6.35 \mathrm{~mm} ; 4.76 \mathrm{~mm} ; 3.18 \mathrm{~mm}$ and $1.50 \mathrm{~mm}$. The wall thickness was chosen to be 3 $\mathrm{mm}$. Different materials were assigned to the different object types in the phantom: skin tissue for the container, glandular tissue as bulk material and adipose tissue for the background spheres. Breast abnormalities included masses and small calcifications, simulated as spherical objects.

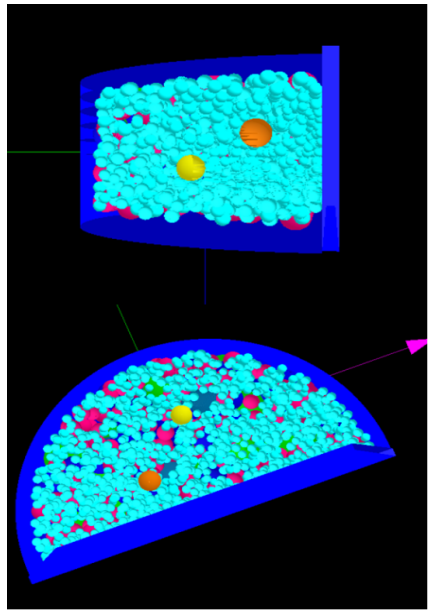

(a)

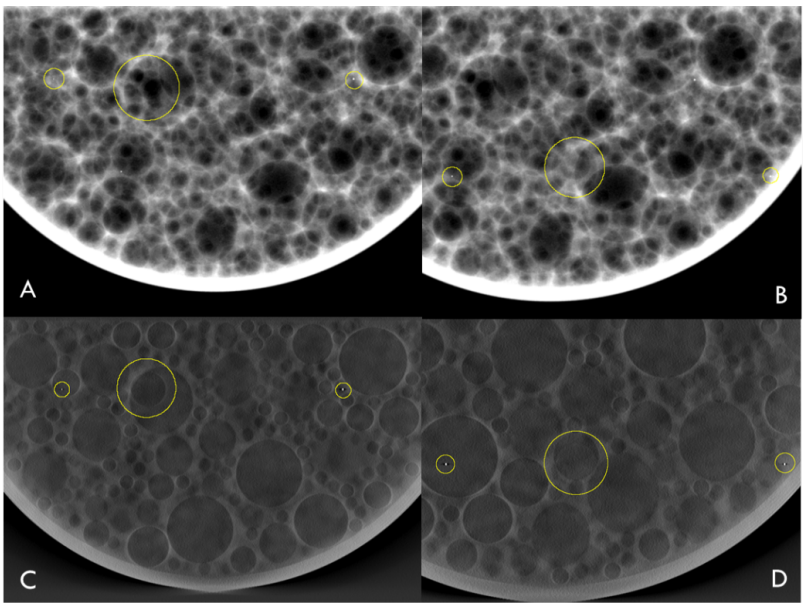

(b)

Figure 9. Work Project Example 2: images are taken from the project work of participants performed the face-to-face phase of the "Anthropomorphic Phantoms" module at the Technical University of Varna, Bulgaria, 7-13 September 2015. (a) generated breast software phantom with the inserted simulated lesions, (b) planar (A and B) and tomosynthesis (C and D) images containing two masses and four small calcifications.

The two masses were simulated as spheres with diameter of $8 \mathrm{~mm}$ and consisted of two different materials $(75 \%$ glandular/25\% adipose and 50\% glandular $/ 50 \%$ adipose tissue). The masses were 
K. Bliznakova, Development of Breast Software Phantom..., Rad. Applic., 2017, 2, 1, 14-19

positioned at two different heights in the phantom. Four calcifications were modelled as small spheres of radii $125 \mu \mathrm{m}, 150 \mu \mathrm{m}, 175 \mu \mathrm{m}$ and $200 \mu \mathrm{m}$ made of $\mathrm{CaCO}_{3}$. They were positioned at the same height in the phantom as the masses. Projection images for beam energy of $20 \mathrm{keV}$ were generated using the XRayImageSimulator and isocentric geometry with source-to-isocenter distance of $600 \mathrm{~mm}$ and source-todetector distance of $650 \mathrm{~mm}$. The detector image size was assigned as $150 \mathrm{~mm} \times 150 \mathrm{~mm}$ with a pixel size of $0.1 \mathrm{~mm}$. For tomosynthesis, the participants acquired 26 projections at different angles ranging from $-25^{\circ}$ to $+25^{\circ}$ with $2^{\circ}$ increment. A Poisson noise distribution was simulated for $8 \mathrm{mGy}$ surface entrance dose and added to the projection images. The 26 tomosynthesis projections were reconstructed into a volume using inhouse developed software tool for reconstructions using the FDK algorithm. Images of both modalities were visually compared and contrast-to-noise ratio was calculated in the images with pixel values consisting of line integrals.

Based on results for the contrast-to-noise ratio and visual observation, participants concluded that small calcifications were equally visible in $2 \mathrm{D}$ mammography and tomosynthesis whereas masses were better visualized and more easily detected in tomosynthesis compared to 2D mammography (Figure $9 \mathrm{~b}$ ).

The above examples showed how the developed software phantom can be used both, for research and educational purposes.

\section{CONCLUSIONS}

The paper demonstrates the successful application and usability of complex and realistic breast software phantoms for breast imaging research and advanced training of Medical Physics Experts.

Future work is related to detail quantitative evaluation of the software phantom and its use in advanced breast imaging research.

Acknowledgement: This work is supported by PHASETOMO Marie Curie Career Integration Grant within the 7 th European Community Framework Programme (PCIGo9-GA-2011-293846) and by EUTEMPE-RX Project of the European Union 7th Framework Program under Grant Agreement No: 605298. The author would like to express her gratitude to the participants of the Anthropomorphic Phantoms EUTEMPE-RX module who provided data for the present work.

\section{REFERENCES}

1. A.-K. Carton, P. Bakic, C. Ullberg, H. Derand, A. D. A. Maidment, "Development of a physical 3D anthropomorphic breast phantom," Med. Phys., vol. 38, no. 2, pp. 891-896, 2011.

DOI: $10.1118 / 1.3533896$

PMid: 21452726 PMCid: PMC4108620

2. L. Cockmartin, N. Marshall, H. Bosmans, "Design and evaluation of a phantom with structured background for digital mammography and breast tomosynthesis," in Proceeding of IWDM'12, Berlin, Heidelberg, Germany: Springer-Verlag, 2012 DOI: $10.1007 / 978-3-642-31271-783$

3. G. J. Gang, D. J. Tward, J. Lee, and J. H. Siewerdsen, "Anatomical background and generalized detectability in tomosynthesis and cone-beam CT," Med. Phys., vol. 37, no. 5, pp. 1948-1965, 2010.

DOI: $10.1118 / 1.3352586$

PMid: 20527529

PMCid: PMC2862054

4. K. Bliznakova, R. Speller, J. Horrocks, P. Liaparinos, Z. Kolitsi, N. Pallikarakis, "Experimental validation of a radiographic simulation code using breast phantom for X-ray imaging," Comput. Biol. Med., vol. 40, no. 2, pp. 208-214, 2010.

DOI: 10.1016/j.compbiomed.2009.11.017 PMid: 20056197

5. EUTEMPE-RX: European Training and Education for Medical Physics Experts in Radiology, in FP7 Fission2013-5.1.1: EURATOM Fission Training Schemes (EFTS) in Nuclear Fission, Safety and Radiation Protection, Project Number: 605298, 01.08.2013 31.07.2016.

6. "Guidelines on Medical Physics Experts", European Commission Radiation Protection Report 174, 2014. 